\title{
A UNIQUENESS THEOREM FOR HOMOLOGY IN Cat, THE CATEGORY OF SMALL CATEGORIES
}

\author{
BY DANA MAY LATCH
}

Communicated by S. Eilenberg, November 27, 1974

I. Introduction. Oberst [7], Laudal [4], Watts [10], and Andre' [1] have shown that derived functors of colimit define a homology theory for Cat, the category of small categories. In this note, we outline a proof of uniqueness for such a homology theory, making extensive use of a Kan-type construction (see e.g. Lemma A) and of uniqueness for homology in $S^{\Delta^{o p}}$, the category of simplicial sets [2]

II. Preliminaries. The following Kan-type construction is used in several contexts.

LEMmA A. Let $\mathrm{C}$ be a cocomplete category, $\mathrm{C}$ a small category, and $\theta$ : $\mathrm{C} \rightarrow \mathrm{C}$ a functor. Then there exists an adjoint pair: the singular functor $S_{\theta}: \mathcal{C}$ $\rightarrow$ Scop defined by $S_{\theta}(A)=\mathcal{C}\left(\theta_{-}, A\right)$, for $A \in|C|$, and its left adjoint $\hat{\theta}:$ Scop $\rightarrow C$.

Let $\Delta$ be the small category whose objects are the finite ordinals $[k]=\{0<$ $1<2<\ldots<k\}$ and whose morphisms are order preserving functions $\mu:[k] \rightarrow$ $[m]$. By considering the full inclusion functor $\iota: \Delta \rightarrow$ Cat, in the context of Lemma A, nerve, $N$ : Cat $\rightarrow S^{\Delta \text { op }}$, is the singular adjoint of categorical realization $c:$ $S^{\Delta}$ op $\rightarrow$ Cat and $c N=\mathrm{id}_{\text {Cat }}[3$, p. 33]. Thus the standard representable $k$-dimensional simplicial set $\Delta[k]$ is actually $N([k])=\Delta(-,[k])$.

Similarly, the functor $\tau: \Delta \rightarrow$ Cat defined as the comma category, $\tau[k]=$ $\Delta \downarrow[k]$, gives rise to another pair of adjoint functors $S:$ Cat $\rightarrow S \Delta$ op and $\Gamma$ : $S^{\Delta}$ op $\rightarrow$ Cat. Let $X \in\left|S^{\Delta}{ }^{\text {op }}\right|$, then $\Gamma X$ is the small category whose objects are $\amalg_{k \geqslant 0} X_{k}$, and whose morphisms are triples $\langle y, \mu, x\rangle$ where $x \in X_{m}$ is the codomain, $\mu:[k] \rightarrow[m]$ in $\Delta$ is the morphism, and $y=X(\mu) x$ in $X_{k}$ is the domain.

The natural transformation "last", $\eta: \tau \stackrel{\bullet}{\rightarrow}$, is given by $\eta_{k}(\alpha:[p] \rightarrow[k])$ $=\alpha(p) \in[k]$. By adjoint functor theory and by the theory of coends, $\eta$ induces

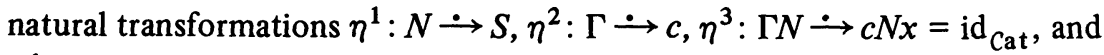
$\eta^{4}: N \Gamma \stackrel{\leftrightarrow}{\rightarrow} \mathrm{id}_{S} \Delta$ op

AMS (MOS) subject classifications (1970). Primary 55 B40; Secondary 18G30, $18 \mathrm{G} 10$.

Key words and phrases. Homology theory, category of small categories, simplicial sets, left derived functors of colimit. 
The Milnor geometric realization functor | |: $S^{\Delta}$ op $\rightarrow$ Top [5], Top the category of $\mathrm{CW}$ complexes, can also be viewed as another example of the "Lemma $\mathrm{A}$ " situation. If $\theta: \Delta \rightarrow$ Top is given by $\theta([k])=\Delta^{k}$, the standard $k$-dimensional affine simplex in $\mathbf{R}^{k+1}$, then |l: $S^{\Delta \text { op }} \rightarrow$ Top is the left adjoint of the singular complex functor $S_{\theta}$ : Top $\rightarrow S^{\Delta}$ op . Let $B=\left|N_{-}\right|:$Cat $\rightarrow$ Top denote the Segal classifying space functor [9]. Then for each small category $\mathbf{C}, B C$ is the $\mathrm{CW}$ complex whose $k$-cells are in one-to-one correspondence with nondegenerate $k$-simplicies of $N C$.

III. Definition of homology and existence. A subcategory $\mathbf{C}^{\prime}$ of $\mathbf{C}$ is initial in $\mathbf{C}$ if all morphisms $m: p \rightarrow q$ in $\mathbf{C}$ with codomain in $\mathbf{C}^{\prime}$ are in $\mathbf{C}^{\prime}$. A pair of small categories $\left(\mathbf{C}, \mathbf{C}^{\prime}\right)$ is said to be admissible if $\mathbf{C}^{\prime}$ is an initial subcategory of $\mathbf{C}$. The category of all admissible pairs and obvious morphisms is also denoted by Cat.

A homology theory for Cat is a pair $\langle h, \partial\rangle$, where $h$ : Cat $\rightarrow A b^{Z}$ is a functor from the category of admissible pairs to graded abelian groups and $\partial_{*}: h_{*}$ $\rightarrow h_{*-1}$ is a natural transformation of degree - 1 , satisfying the standard Eilenberg-Steenrod-Milnor axioms [6] : dimension, exactness, excision, homotopy and strong additivity. We state the last three below.

ExCISION AXIOM. Let $\mathbf{C}$ be any small category with initial subcategories $\mathbf{C}_{1}$ and $\mathbf{C}_{2}$. Then the inclusions induce $h_{*}\left(\mathbf{C}_{2}, \mathbf{C}_{1} \cap \mathbf{C}_{2}\right) \cong h_{*}\left(\mathbf{C}_{1} \cup \mathbf{C}_{2}, \mathbf{C}_{1}\right)$, where $\mathbf{C}_{1} \cap \mathbf{C}_{2}$ and $\mathbf{C}_{1} \cup \mathbf{C}_{2}$ are subcategories of $\mathbf{C}$ making the following square bicartesian in Cat:



Номотору АХІом. If $F: \mathbf{C} \rightarrow \mathbf{D}$ is a weak homotopy equivalence, i.e. if $B F: B C \rightarrow B \mathrm{D}$ is a homotopy equivalence in Top, then $h_{*} F: h_{*} \mathrm{C} \rightarrow h_{*} \mathrm{D}$ is an isomorphism.

Strong Additivity (Milnor) AXIOM. Let $\left\{\left(\mathbf{C}_{\alpha}, \mathbf{C}_{\alpha}^{\prime}\right) \mid \alpha \in A\right\}$ be a collection of admissible pairs in Cat. Then the inclusions induce

$$
\bigoplus_{\alpha} h_{*}\left(\mathbf{C}_{\alpha}, \mathbf{C}_{\alpha}^{\prime}\right) \cong h_{*}\left(\underset{\alpha}{\operatorname{II}} \mathbf{C}_{\alpha}, \underset{\alpha}{\amalg} \mathbf{C}_{\alpha}^{\prime}\right)
$$

We assume that the coefficient group $A \in A b$ is fixed. More general coefficient systems will be discussed in the longer exposition.

Define $\Delta_{\mathrm{C}, \mathrm{C}^{\prime}}(A): \mathrm{C} \rightarrow \mathrm{Ab}$ by 


$$
\Delta_{\mathrm{C}, \mathrm{C}^{\prime}}(A)_{p}= \begin{cases}A & \text { if } p \notin\left|\mathbf{C}^{\prime}\right|, \\ 0 & \text { otherwise }\end{cases}
$$

on objects and in the obvious fashion on morphisms.

REMARK. $\mathbf{C}^{\prime}$ initial in $\mathbf{C}$ guarantees that $\Delta_{\mathrm{C}, \mathrm{C}^{\prime}}(A)$ is a functor. Other pairs, e.g., $\mathbf{C}^{\prime}$ terminal in $\mathbf{C}$ or $\mathbf{C}^{\prime}$ an "interval" in $\mathbf{C}$ would also satisfy this condition.

THEOREM 1. 〈H, $\partial\rangle$ is a homology theory for Cat, where

$$
H_{*}\left(\mathbf{C}, \mathbf{C}^{\prime}\right)=L_{*} \operatorname{colim}\left(\Delta_{\mathbf{C}, \mathbf{C}^{\prime}}(A)\right) \text {, }
$$

$L_{*}$ colim: ${ }_{\mathrm{C}} \mathrm{Ab} \mathrm{b}^{\mathrm{C}} \rightarrow \mathrm{Ab}$ being the left derived functors of colim $\mathrm{C}_{\mathrm{C}}: \mathrm{Ab}^{\mathrm{C}} \rightarrow \mathrm{Ab}$.

Proof. See [7] and [4].

Using the canonical coflabby resolution of $\Delta_{\mathrm{C}, \mathrm{C}^{\prime}}(A)([10],[4],[7])$ we see that the complexes used to calculate the homology yield the following:

COROLlARY. Let $\langle H, \partial\rangle$ be the unique homology in $S^{\Delta}$ op [2], i.e. singular homology. Then

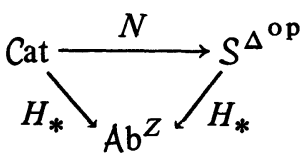

commutes up to isomorphism.

IV. Uniqueness. The proof of our uniqueness theorem rests on the following two lemmas both of which are used in applying the homotopy axiom.

LEMma B. The natural transformation $\eta^{4}: N \Gamma \rightarrow \mathrm{id}_{S^{\Delta}}$ op induces a natural homotopy equivalence $\left|\eta_{X}^{4}\right|:|N \Gamma X| \rightarrow|X|$ in Top, for every simplical set $X$.

LEmma C. The natural transformation $\eta^{3}: \Gamma N \stackrel{\rightarrow}{\rightarrow} \mathrm{id}_{\text {Cat }}$ is a weak homotopy equivalence in Cat, i.e. $\eta^{3}: \Gamma N \mathrm{C} \rightarrow \mathrm{C}$ is a weak homotopy equivalence for each small category $\mathbf{C}$.

THEOREM 2. If $\langle h, \partial\rangle$ is a homology theory for Cat, then

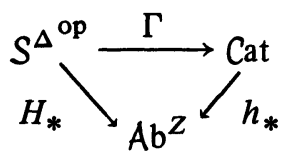

commutes up to isomorphism. 
The proof consists of showing that $h_{*} \Gamma: S^{\Delta}$ op $\rightarrow \mathrm{Ab}^{Z}$ satisfies the standard axioms for homology theory in $S^{\Delta}$ op . Hence, by uniqueness of such a theory [2] , the diagram commutes. Some of the special properties of $\Gamma: S^{\Delta \text { op }} \rightarrow$ Cat used in the proof are that $\Gamma$ commutes with pullbacks, $\Gamma(\Delta[k])$ is contractible, and $\left(\Gamma X, \Gamma X^{\prime}\right)=\Gamma\left(X, X^{\prime}\right)$ is an admissible pair. Lemma $\mathrm{B}$ is needed in proving the homotopy axiom.

THEOREM 3 (Uniqueness). If $\langle h, \partial\rangle$ is a homology theory for Cat then $h_{*}\left(\mathbf{C}, \mathbf{C}^{\prime}\right) \cong H_{*}\left(\mathbf{C}, \mathbf{C}^{\prime}\right)$.

Proof. $h_{*}\left(\mathbf{C}, \mathbf{C}^{\prime}\right) \cong h_{*}\left(\Gamma N \mathrm{C}, \Gamma N \mathbf{C}^{\prime}\right)$ by the homotopy axiom used in conjunction with Lemma C. By Theorem $2, h_{*}\left(\Gamma N C, \Gamma N C^{\prime}\right) \cong H_{*}\left(N C, N C^{\prime}\right)$. But the Corollary guarantees that $H_{*}\left(N \mathrm{C}, N \mathrm{C}^{\prime}\right) \cong H_{*}\left(\mathbf{C}, \mathbf{C}^{\prime}\right)$. Q.E.D.

ACKNOWLEDGEMENTS. I would like to express my appreciation for many fruitful conversations with A. Heller and J. Milnor.

\section{BIBLIOGRAPHY}

1. M. André, Méthode simpliciale en algèbre homologique et algèbre commutative, Lecture Notes in Math. Vol. 32, Springer-Verlag, Berlin and New York, 1967. MR 35 \#5493.

2. Yuh-ching Chen, Stacks, costacks, and axiomatic homology, Trans. Amer. Math. Soc. 145 (1969), 105-116. MR 40 \#3536.

3. P. Gabriel and M. Zisman, Calculus of fractions and homotopy theory, Ergebnisse der Mathematik und ihrer Grenzgebiete, Band 35, Springer-Verlag, New York, 1967. MR 35 \#1019.

4. O. A. Laudal, Sur les limites projectives et injectives, Ann. Sci. École. Norm. Sup.

(3) 82 (1965), 241-296. MR 34 \#225.

5. J. Milnor, The geometric realization of a semi-simplicial complex, Ann. of Math.

(2) 65 (1957), 357-362. MR 18, 815.

6. On axiomatic homology theory, Pacific J. Math. 12 (1962), 337-341. MR 28 \#2544.

7. U. Oberst, Homology of categories and exactness of direct limits, Math. Z. 107 (1968), 87-115. MR 39 \#5662.

8. J.-E. Roos, Sur les foncteurs dérivés de lim. Applications, C.R. Acad. Sci. Paris 252 (1961), 3702-3704. MR 24 \#A1938.

9. G. Segal, Classifying spaces and spectral sequences, Inst. Hautes Études Sci. Publ. Math. No. 34 (1968), 105-112. MR 38 \#718.

10. C. E. Watts, $A$ homology theory for small categories, Proc. Conf. on Categorical Algebra (LaJolla, Calif. 1965), Springer, New York, 1966, pp. 331-335. MR 34 \#4336.

\section{DEPARTMENT OF MATHEMATICS, RUTGERS UNIVERSITY, NEW BRUNSWICK, NEW JERSEY 08903}

Current address: Department of Mathematics, Lawrence University, Appleton, Wisconsin 54911 